\title{
La eutanasia y la medicalización de la muerte desde una perspectiva jurídica compleja
}

\section{Euthanasia and the medicalization of death \\ from a complex juridical perspective}

\author{
A eutanásia e a medicalização da morte a \\ partir de uma perspectiva jurídica complexa
}

Fecha de recepción: 12 de abril de 2016

Fecha de evaluación: 15 de noviembre de 2016

Fecha de aceptación: 31 de julio de 2017

Disponible en línea: 21 de noviembre de 2017

Elvio Galati*

\begin{abstract}
Cómo citar:
Galati, E. (2018). La eutanasia y la medicalización de la muerte desde una perspectiva jurídica compleja. Revista Latinoamericana de Bioética, 18(1), 68-86.

Doi: https://doi.org/10.18359/rlbi.1833
\end{abstract}

* $\quad$ Posdoctor en Derecho. Profesor en Ciencias Jurídicas, profesor asociado de Derecho de la Salud, Ética Profesional e Introducción al Pensamiento Científico de la Universidad Abierta Interamericana (UAI) y docente regular de la Universidad Nacional de Rosario (UNR). Director de un proyecto de investigación, del Centro de Altos Estudios en Ciencias Humanas y de la Salud (CAECIHS), Rosario, Argentina, e investigador científico 1b de la Universidad Abierta Interamericana. Correo electrónico: elviogalati@gmail.com. ORCID: https://orcid. org/0000-0003-3783-2479. 
Este trabajo trata el fenómeno de la medicalización de la vida relacionado con el fin de esta; esboza, además, vínculos con el derecho a través de la juridización de la salud, y así incorpora como resultado este término. Se hace hincapié en un caso actual sobre retiro del soporte vital y se relaciona con la ley de derechos del paciente, fallos, legislación extranjera y doctrina médica, bioética y jurídica. El marco teórico es el del pensamiento complejo de Edgar Morin y la teoría trialista del mundo jurídico. El aporte se centra en el tratamiento complejo y trialista del fenómeno de la medicalización de la muerte. En cuanto a la metodología, se plantea la cualitativa, estudiando casos reflejados en fallos, y analizando su discurso. El tema permite tratar problemas de teoría general del derecho como el vacío legislativo y el papel del juez. Se concluye provisoriamente que Argentina tiene una cultura juridizadora, a pesar de la ley que contempla la llamada muerte digna.

Palabras clave: complejidad, medicalización, muerte, salud, juridización, eutanasia.

\section{Abstract}

The paper deals with the phenomenon of the medicalization of life, related to the end of it, outlining links with the law through the legalization of health, and thus incorporates this term as a result. It focuses on a current case of withdrawal of life support and it's related to the patient's rights law, judgments, international legislation, and medical, bioethical and legal doctrine. The theoretical framework is the complex thought of Edgar Morin and the trialist theory of the legal world. The contribution focuses on the complex and trialist treatment of the phenomenon of the medicalization of death. As for the methodology, the qualitative one is raised, studying cases reflected in failures, and analyzing its speech. The subject allows dealing with problems of general theory of law such as the legislative vacuum and the role of the judge. It is provisionally concluded that Argentina has a legal culture, despite the law that includes for the so-called dignified death.

Keywords: Complexity, medicalization, death, health, legalization, euthanasia.

\section{Resumo}

Este trabalho trata o fenômeno da medicalização da vida relacionado com o fim dela; ele também descreve os laços com o direito através da juridicização da saúde, e assim incorpora esse termo como resultado. Faz-se ênfase em um caso atual sobre a retirada do suporte vital e está relacionado com a lei dos direitos do paciente, falhas, legislação estrangeira e doutrina médica, bioética e jurídica. O quadro teórico é o do pensamento complexo de Edgar Morin e a teoria trialista do mundo jurídico. A contribuição centra-se no tratamento complexo e trialista do fenômeno da medicalização da morte. Quanto à metodologia, apresenta-se a qualitativa, estudando casos refletidos em falhos e analisando seu discurso. O tema permite lidar com problemas de teoria geral do direito, como o vácuo legislativo e o papel do juiz. Conclui-se provisoriamente que a Argentina tem uma cultura juridicizadora, apesar da lei que prevê a chamada morte digna.

Palavras-chave: complexidade, medicalização, morte, saúde, juridicização, eutanásia. 


\section{Introducción}

En este artículo se apunta a desarrollar la medicalización de la vida en relación con el final de esta, abordando este fenómeno desde el pensamiento jurídico complejo. Con este marco teórico el derecho es visto en tres dimensiones: social, normativa y valorativa, lo que amplía el análisis y permite que se gane en riqueza, completud, transparencia y profundidad.

La medicalización de la vida hace referencia a la intervención de la medicina en temas que deberían dejarse al amparo de otras ramas de la ciencia o a lo que en gnoseología se conoce como el conocimiento popular, siendo este un aspecto de la llamada cientifización de la vida. Literalmente significa transformar en médico un problema que no lo es (Natella, 2008). El origen de este término puede encontrarse en Iván Illich (1975), que señaló que la medicalización apunta a "[...] expropiar el poder del individuo para curarse a sí mismo y para modelar su ambiente" (p. 9). De esta forma, "[...] denota la influencia de la medicina en casi todos los aspectos de la vida cotidiana [...]" (Mainetti, 2006, p. 72). Esto se da en el caso de la calvicie, el vigor sexual, el control del peso, estado de ánimo, la postergación del envejecimiento (Salamano, 2007), el día femenino, el acné, las cirugías estéticas, etc. "Hay sobreconsumo de productos cuyas virtudes son exageradas o ilusorias, que prometen salud, belleza, longevidad, rejuvenecimiento, virilidad" (Morin, y Hessel, 2013, p. 54). También se da cuando se convierten meros factores de riesgo en enfermedades, como ocurre con el colesterol ${ }^{1}$ o la osteoporo-

1 "[...] si mejorar la salud humana fuera nuestro principal objetivo, algunos de los miles de millones invertidos actualmente en los costosos fármacos para reducir el nivel de colesterol de la gente sana serían mucho más rentables si se gas- $\operatorname{sis}^{2}$. Es decir, "la realización indiscriminada de actividades de cribado, las revisiones repetidas por factores de riesgo, etc. acaban convirtiendo a personas sanas en enfermos dependientes del sistema" (Orueta Sánchez, Santos Rodríguez, González Hidalgo, Fagundo Becerra, 2011, p. 211). Un ejemplo de medicalización se ve cuando el papa Francisco distribuye envasada como medicamento a la "misericordia", definida por la Real Academia Española como "atributo de Dios, en cuya virtud perdona los pecados y miserias de sus criaturas". Lo anterior da a entender que el lenguaje ordinario se ha medicalizado (Mainetti, 2006, p. 78). Otro ejemplo se ve cuando un estudio etnológico de un hospital parisino señala que después de diez años de servicio un auxiliar de enfermería tiene mejor sentido común que los enfermeros y médicos: se pueden adaptar mejor al enfermo, saben cuándo va a morir y se ocupan no solo de la enfermedad, sino también de su estado psicológico (Jolicorps, 2007, 10). Es inherente a la medicalización erigirse en un estadio superior desde

taran en campañas contra el tabaquismo, o para aumentar la actividad física y mejorar la alimentación" (Moynihan y Cassels, 2006, p. 14).

2 En el caso del tratamiento de la osteoporosis, a través del fármaco Fosamax, se hizo un estudio que mostró que dicho fármaco reducía el riesgo de fracturas de caderas en un $50 \%$. Pero en ese estudio solo se incluyeron mujeres con alto riesgo, es decir, mujeres mayores, que ya habían sufrido al menos una fractura, cuando el grupo al que se dirigía el fármaco era mucho más grande, es decir, decenas de millones de mujeres sanas, que sufrían la promoción de los tests de medición de la densidad ósea (Moynihan y Cassels, 2006, p. 153). Lo que en realidad puede prevenir las fracturas de cadera son los programas que tratan de evitar los eventos traumáticos que suelen preceder a la fractura, como la ingesta múltiple de medicamentos en los mayores, fijar las alfombras a los suelos, las mejoras en la visión; porque relajarse en el remedio evita tomar dichas medidas preventivas (Moynihan y Cassels, 2006, p. 154). 
el cual se "prescribe" al enfermo lo que debe hacer. En efecto, "[...] no toda entidad que es tratada como una enfermedad es un problema médico" (Epele, 2008). Ver la vida a través de los ojos de la ciencia es reducirla.

Por su parte, siguiendo el esquema de la medicalización, la juridización implica que el derecho aborda o regula temas que no debería tratar. De ahí que tanto la medicalización, como la cientifización y la juridización, en este caso de la salud, no sean tributarias de un pensamiento complejo; por el contrario, son elementos propicios para reducir el todo a una parte de la vida. La medicalización reduce el tratamiento de los problemas sanitarios a su aspecto biológico, con lo cual la salud, como estado de bienestar compuesto por aspectos biológicos, psicológicos, sociales, espirituales, es solo abarcada, tratada con una pastilla; de la misma manera que es reducida la vida a la lógica de la máquina artificial (Morin, 1984, p. 80).

\section{[...] la complejidad es la unión de la simpli- cidad y de la complejidad; es la unión de los procesos de simplificación que implican se- lección, jerarquización, separación, reduc- ción, con los otros contra-procesos que im- plican la comunicación, la articulación de aquello que está disociado y distinguido; $\mathrm{y}$ es el escapar de la alternativa entre el pen- samiento reductor que no ve más que los elementos y el pensamiento globalista que no ve más que el todo. (Morin, 2005, p. 144)}

Reducir el análisis será entonces "artificializar" la vida jurídica, sumirla en un mundo de laboratorio que no tendrá correspondencia con la realidad que le tocará vivir al profesional del derecho. Nótese que la normatividad lleva en sí una fuerte referencia a la abstracción y una de las tentaciones racionalistas es la normalización, "[...] es decir, eliminar y combatir lo extraño, lo irreductible y el misterio" (Morin, Ciurana, Motta, 2002, p. 26). Al contrario, "[...] el problema del método consistía en su posible aptitud para capturar lo efímero, lo contingente, la novedad, la multiplicidad, en fin, la complejidad" (Morin et al., 2002, p. 20).

Justamente, una de las formas de la eutanasia, nombre tradicional y técnico que aborda las problemáticas alrededor de la muerte, es el encarnizamiento terapéutico, es decir, el empleo de medios extraordinarios sin base humana que los justifique, porque para el caso son innecesarios. "Un porcentaje creciente de fondos procedentes de nuevos impuestos se destina a medios tecnológicos para prolongar la vida de pacientes en estado terminal" (Illich, 1975, p. 62). La muerte no está compuesta solamente por procesos biológicos, sino también por aspectos culturales, sociales, espirituales, y se requiere contacto con el mundo, comunicación, relación con seres queridos, y su participación en la muerte de una persona, así como participan en la vida. Aquí se notan los distintos aspectos, dimensiones que abarcan un fenómeno captado en su conjunto. La "[...] complejidad [...] es [...] un tejido de constituyentes heterogéneos inseparablemente asociados, que presentan la paradójica relación de lo uno y lo múltiple" (Morin et al., 2002, p. 40).

Cabe preguntarse qué respuesta da el derecho frente a este fenómeno, a fin de no transformarse en juridizador de la salud, es decir, permitiendo la intervención innecesaria del Estado en el ámbito del final de la vida. Para esto, complejizando el análisis jurídico, incorporaremos el estudio de los aspectos sociales, normológicos y valorativos, según el esquema de la teoría trialista del mundo 
jurídico, que plantea en la disciplina jurídica todas esas dimensiones (Galati, 2012).

\section{Aspecto jurístico-sociológico}

Es importante cuestionarse quién es el encargado de adjudicar vida y muerte. Pasada la Edad Media debimos aprender la lección de la pérdida del poder de Dios para decidir en ese ámbito. Después de la Edad Moderna debimos aprender la lección de la pérdida del poder de la ciencia médica para decidir en ese ámbito. Posterior a la Edad Contemporánea debimos aprender de la lección de la muerte de Dios y de las consecuencias ambivalentes de la ciencia. No obstante, el mercado y la tecnociencia asumen las formas de opresión del individuo, si es él quien tiene que decidir, y restan aún resabios clericales si se observa a quién va dirigida una carta de Ramón Sanpedro (2005): "[...] es miembro del Opus Dei. Me acusa de ser tan soberbio que me quiero parecer a Dios por reclamar el derecho a disponer de mi vida" (p. 239). ¿Es libre el individuo para decidir? La medicalización implica la pérdida del poder del individuo para decidir en su salud. Foucault encuadra filosóficamente dicho afán uniformizante en la normalización vinculando a la medicina con el derecho. Así,

[...] en el siglo XX los médicos están inventando una sociedad, ya no de la ley, sino de la norma. Los que gobiernan en la sociedad ya no son los códigos sino la perpetua distinción entre lo normal y lo anormal, la perpetua empresa de restituir el sistema de la normalidad. (Foucault, 1996, p. 76)

Lo anterior guarda relación con la lógica de la máquina artificial de la que hablábamos.
El convidado de piedra, todavía hoy, es el enfermero, quien tiene un contacto personal con el muriente, y es más sensible a la situación dolorosa, lo cual favorece que se opongan a quienes prescriben en los casos en los que se sufre inútilmente (Jolicorps, 2007, p. 8), es decir, cuando se medicaliza. No por casualidad se habla de la falta de humanización de la medicina, precisamente por la falta de contacto con el ser humano, el muriente en este caso. En efecto, un manual de ética para el personal de enfermería señala: "se deberán substituir los medicamentos por palabras y gestos, siendo la humanidad del personal, más que la ciencia quien actúe" (Valls Molins, 1993, p. 289).

Una manera de plantear la autonomía del paciente, reafirmando su señorío, es el de las directivas anticipadas, que son aquellas "instrucciones" que deja quien tenía consciencia al momento de redactarlas, para el eventual caso en el que se dé el supuesto eutanásico sumado al de ausencia de la consciencia, a fin de encaminar el estado de salud del paciente. Si no hay directivas, se vuelve sobre el problema de la legitimación. Cabe traer a colación lo expresado por un juez en el caso "S., M. de C. s/ insania" de febrero de 2005, referido al esposo devenido curador que pretendía que se suspendiera la hidratación y nutrición artificiales ?por sonda?, que se venía aplicando por varios años (más de 5 años) a una mujer que padecía estado vegetativo permanente, como consecuencia de trastornos posparto que derivaron en una encefalopatía hipóxica con convulsiones generalizadas y con pronóstico de irreversibilidad. Eso implicaba una desconexión cognitiva absoluta e irreversible, y ausencia completa de toda actividad consciente. La Corte de la Provincia de Buenos Aires decidió en febrero de 2005 denegar el pedido. Señala el juez Hitters: 
[...] corresponde al enfermo -y sus familiares- juzgar si el empleo de instrumento y personal es desproporcionado a los resultados previsibles, y si las técnicas usadas imponen al paciente mayores sufrimientos y molestias que los beneficios que se puedan obtener de los mismos. Se dice entonces que ante la inminencia de la muerte inevitable, a pesar de los medios empleados, es lícita en conciencia la decisión de renunciar a tratamientos que procurarían únicamente una prolongación precaria y penosa de la existencia [...]. (Consid. II)

Plantear la medicalización en el final de la vida implica preguntarse por el encargado de decidirla. En efecto, las directivas hacen referencia a la libertad de cada uno de tomar sus decisiones, para que su vida sea conforme a sus deseos (Jolicorps, 2007, p. 5).

Por otro lado, vale la pena preguntarse por lo que está en juego en esta temática, es decir, en qué consiste la vida, un tratamiento terapéutico. Precisamente, un médico, Jorge Manzini (1997), fundamenta la insuficiencia de la medicina a la hora de abordar el problema de la eutanasia: "[...] los tratamientos de soporte vital sostienen únicamente la dimensión biológica de la vida" (p. 140), y se pregunta por la naturaleza de la salud, señalando "[...] si debe considerarse tratamiento médico aquel procedimiento del que no puede esperarse que produzca ninguna modificación del cuadro, sino sólo un mantenimiento del 'status quo'" (p. 140). Precisamente, la complejidad apunta a tratar todas las dimensiones de un problema y, en este caso, no solamente la dimensión biológica de la vida.

La medicalización de la vida, en el final de ella, implica perjuicios de todo tipo, porque se emplean recursos sanitarios donde no se los deberían emplear, ya que se pretende mantener una vida desde el punto de vista biológico, en tanto se pierde el contacto con el mundo a través de la falta de consciencia, o se sabe que el pronóstico de muerte es inexorable, lo que impacta en el estado espiritual del paciente y de su familia.

Asimismo, hay que cuestionarse cuándo hay medicalización, es decir, cuándo quienes dicen brindar salud abusan de sus conocimientos con el pretexto de curar. El fin de la vida y la eutanasia, y el auxilio al suicidio plantean formas de terminar la vida, es decir, caminos para llegar a una solución, lo que en el derecho se categoriza como la forma de la conducta. Aquí pueden verse, por ejemplo, las distintas formas de la eutanasia o las clasificaciones clásicas. Se trata de la muerte piadosa de enfermos terminales que desean concluir con los dolores y angustias propias de una dolencia o agonía, que tiene variantes: eutanasia pasiva o eliminación de los medios médicos para prolongar la vida, eutanasia activa directa, o suministro al enfermo de un tratamiento que le produce la muerte, y la activa indirecta, en la cual se le proporciona al paciente un calmante que, además de aliviarlo, le puede ocasionar la muerte (Sagüés, 1999, p. 326). Se hablará de distanasia cuando hay una obstinación o encarnizamiento terapéutico, es decir, el empleo de medios injustificados o desproporcionados en relación con el estado de la persona, porque no contribuyen a la mejoría de su salud, son inútiles. En la eutanasia activa se emplean medios comisivos para procurar la muerte de una persona que se halla en estado de gravidez por tener una enfermedad irreversible, sin pronóstico favorable y padeciendo dolores insoportables. Estos son los requisitos para la eutanasia que establece la pionera ley holandesa 26691, de mayo de 2001, sobre "comproba- 
ción de la terminación de la vida a petición propia y del auxilio al suicidio". Allí se expone un capítulo dedicado al "cuidado y esmero profesional", donde el médico debe comprobar que "[...] la petición del paciente es voluntaria y bien meditada", que "[...] el padecimiento del paciente es insoportable y sin esperanzas de mejora", que se ha informado de "[...] la situación en que se encuentra y de sus perspectivas de futuro", y que se ha "[...] llegado al convencimiento junto con el paciente de que no existe ninguna otra solución razonable para la situación".

Se requiere también la consulta con otro médico independiente que ha visto al paciente y que ha emitido su dictamen sobre el cumplimiento de los requisitos de cuidado (artículo 2). Luego, una comisión comprobará si el médico ha actuado conforme a dichos requisitos; de no ser así, comunicará la situación a la Fiscalía General del Estado y al inspector regional para la asistencia sanitaria (artículos 8 y 9). Puede ser pasiva u ortotanasia, cuando a los requisitos de base se suma la omisión de aquellos que pudiendo hacer "algo" para procurar el restablecimiento de la vida de la persona no lo hacen. En la eutanasia pasiva, las medidas extraordinarias o desproporcionadas representan un riesgo para el enfermo y le producen incomodidad, dolor o sufrimiento, y por ello se consideran inútiles al no esperarse respuesta favorable alguna, lo que no impide continuar con las medidas paliativas del dolor (Gutiérrez-Samperio, 2001, p. 272).

Surge también la pregunta acerca de las razones de las conductas. Hablando de las sociales, es decir, de la opinión que la sociedad tiene sobre el tema, es una tarea difícil, pero no imposible de analizar. Sí, estoy seguro de que la sociedad tiene participación para opinar por cuanto se trata de recursos públicos que se aplican en materia sanitaria. No debería abusar la sociedad de su participación, puesto que en muchos casos resonantes, como el de Karen Quinlan, Camila Sánchez o Marcelo Diez, se filtra información privada y suele haber un desborde del juicio, lo que permite abusos en la opinión o violaciones a la intimidad de los involucrados. ${ }^{3}$

Así pues, hay distintas maneras de ordenar las conductas, más horizontalmente, donde todos participan espontáneamente y, más verticalmente, donde unos dicen a otros lo que tienen que hacer, planificadamente. En el tratamiento del final de la vida se hace referencia a un "orden de realidad causal", del cual serían tributarios los médicos, tratando de encontrar las causas de la enfermedad y de erradicar sus consecuencias por los tratamientos, dejando de lado la experiencia de vida y el parecer de aquellos que viven la enfermedad y rodean al enfermo. El médico se focaliza sobre un punto, por ejemplo, un problema agudo potencialmente reversible, como el mejoramiento de la función real, y en esa escalada olvida el contexto no médico. La consideración del contexto es una idea vital de la complejidad: "[...] un saber no es pertinente si no es capaz de situarse dentro de un contexto, y [...] el conocimiento más sofisticado, si está totalmente aislado, deja de ser pertinente [...]" (Morin, 2007,

3 Sobre el caso de "Marcelo Diez" p. v. Maglio, I. (2012). Dejen ir a Marcelo en paz. Obtenida el 28 de noviembre de 2013 de http://www.rionegro.com.ar/diario/dejen-ir-a-marcelo-en-paz943988-9539-nota.aspx; Iglesias, M. (2012). Ahora piden que 'dejen ir' a Marcelo. Recuperado de http://www.clarin.com/sociedad/Muerte-ahora-piden-dejen-Marcelo_0_728327332.html; S/A (2013). El papa Francisco ya estudia el caso Marcelo Diez (ver: http://www.neuquen.com. ar/sociedad/13890-el-papa-francisco-ya-estudia-el-caso-marcelo-diez.html). 
p. 91). Y el "orden de realidad participativo", del cual serían tributarios los enfermeros, que consultan al paciente y su entorno para adaptar mejor los cuidados, que negocian con los médicos los tratamientos, y son el obstáculo al encarnizamiento terapéutico (Jolicorps, 2007, p. 9). La participación hace referencia al involucramiento del paciente y los interesados en la situación sanitaria de que se trate. Cuando la complejidad menciona al contexto, vuelve a hacer referencia a la multiplicidad ontológica: "el contexto abarca todo ente de la realidad, desde lo material, pasando por lo inmaterial (ideas, sentimientos) hasta lo desconocido. [...] Nada está desconectado [...]" (Morin, 2007, pp. 24 y 25).

No está reflejado en las normas, pero un estudio señala que más se sube en la escala jerárquica y menos tiempo se pasa con el enfermo (Jolicorps, 2007, 10). "On remarque ainsi qu'être proche du malade va de pair avec un statut hiérarchique inférieur ${ }^{4 \prime \prime}$ (Jolicorps, 2007, p. 11).

\section{Aspecto jurístico-normológico}

La eutanasia activa directa está generalmente reprimida por los Estados (Sagüés, 1999, p. 327), salvo los casos de Holanda y Suiza, por ejemplo. En Argentina está prohibida, y solo se admite la eutanasia pasiva, que se conoce bajo el nombre de muerte digna, debido al estigma que tiene la palabra en la academia y la población. No es el caso de otros lugares donde incluso está, por ejemplo, la Asociación por el Derecho de Morir en Dignidad, de Suiza (Jolicorps, 2007, p. 5). En Argentina se reformó la ley de derechos del paciente, 26529, y en la parte de la "autonomía de la volun-

4 "Se nota que estar cerca del enfermo va de la mano con un estatus jerárquico inferior" (trad. del autor). tad" se lee: "El paciente tiene derecho a aceptar o rechazar determinadas terapias o procedimientos médicos o biológicos, con o sin expresión de causa, como así también revocar posteriormente su manifestación de la voluntad" (artículo 1 de la Ley 26742). El supuesto de hecho de la norma es similar al de la ley holandesa: "[...] el paciente que presente una enfermedad irreversible, incurable o se encuentre en estado terminal, o haya sufrido lesiones que lo coloquen en igual situación, informado en forma fehaciente [...]" (artículo 1). Seguidamente, la ley argentina prevé la eutanasia pasiva u ortotanasia, cuando señala que el paciente "también podrá rechazar procedimientos de hidratación o alimentación cuando los mismos produzcan como único efecto la prolongación en el tiempo de ese estadio terminal irreversible o incurable" (artículo 1), y la distanasia o prohibición del encarnizamiento terapéutico al decir que el paciente "[...] tiene el derecho a manifestar su voluntad en cuanto al rechazo de procedimientos quirúrgicos, de reanimación artificial o al retiro de medidas de soporte vital cuando sean extraordinarias o desproporcionadas en relación con la perspectiva de mejoría, o produzcan un sufrimiento desmesurado" (artículo 1). Todo esto se repite cuando la ley trata las condiciones del consentimiento informado (artículo 2). Pero en ambos casos se prevé la administración de los llamados cuidados paliativos. Dice la ley: "En todos los casos la negativa o el rechazo de los procedimientos mencionados no significará la interrupción de aquellas medidas y acciones para el adecuado control y alivio del sufrimiento del paciente" (artículo 1), lo que luego es repetido al hablar del consentimiento informado, para prever "el derecho a recibir cuidados paliativos integrales en el proceso de atención de su enfermedad o padecimiento" (artículo 2). Los cuidados paliativos consisten en "[...] 
el alivio del sufrimiento y el logro de la mayor calidad de vida posible hasta la muerte, para el paciente y su familia. Y el apoyo para la elaboración del duelo en los sobrevivientes" (Manzini, 1997, p. 35). No buscan binariamente aferrarse a la vida ni acercar la muerte, sino el confort en los últimos momentos de la persona, en el final de su vida. El rechazo al binarismo es otra idea fuerte del pensamiento complejo: "[...] hay que evitar encerrarse en un pensamiento binario, vale decir, un pensamiento obnubilado por un solo polo de atención, en detrimento de los otros" (Morin, 2006, p. 105).

Si la medicalización se da en el ámbito de la dimensión sociológica, la juridización encuentra terreno propicio en la dimensión normológica. La juridización será "originaria" cuando el derecho haya abordado indebidamente el fenómeno sin que la medicina lo haya hecho previamente mediante la medicalización, y "derivada" cuando el derecho trata jurídicamente un caso que ya fue medicalizado. Por ello, hablar de la medicalización de la vida en la dimensión normológica es hablar de juridización, que puede tomar carriles judiciales, administrativos o legales, según el departamento del Estado que formalice: mediante judicialización, burocratización o legalización.

La ley holandesa sobre eutanasia no es juridizadora, por cuanto deja el tratamiento del tema en manos de expertos, es decir, de aquellos que tienen el conocimiento científico y artístico necesario para llevar a cabo la terminación de la vida con el cuidado del caso. Incluso, el Estatuto del Colegio de Médicos de la Provincia de Santa Fe (Ley 3950) menciona tres veces al "arte de curar", lo que muestra que no todo es técnico. Se señalan como componentes de la medicina al arte clínico, la ética médica y las ciencias básicas que le dan sustento (Coic G., 2008, p. 509), y en la medida que se amplían las dimensiones o aspectos del tema, se lo complejiza. El involucramiento del "arte" es otro signo de ausencia de juridización, pues tratándose con seres humanos, hay que incorporar a la decisión y el procedimiento el aspecto humano, dado por la buena comunicación, el cuidado paliativo, las figuras "científicas" del arte de curar. Nótese que el cuidado del paciente es el objetivo de la enfermería (Egúsquiza Pozo, 2005, pp. 33, 44, 54). La Ley santafesina 4931 estatuye el "Código de Ética de los Profesionales del arte de curar y sus ramas auxiliares". A pesar de esto, señala la ley: "En ningún caso el médico está autorizado para abreviar la vida del enfermo, sino para aliviar su enfermedad, mediante los recursos terapéuticos del caso" (artículo 128).

Toda totalización es un yerro, por cuanto nada hay absoluto, y más desde la consciencia de la relatividad, proveniente de la madre de las ciencias: la física. Paul Feyerabend (2003) señala que no hay, por un lado, "actividades puramente científicas" (p. 10); por otro lado, tampoco hay "actividades puramente artísticas" (p. 10), y sí en el centro, una zona indefinida donde se mezclarían esos dos ámbitos. Se ve, por ejemplo, la intervención del arte en la ciencia a la hora de las invenciones positivas y sorprendentes (Feyerabend, 2003, p. 10). No hay que olvidar que las disciplinas actuales fueron en su origen "artes", difiriendo por sus resultados, antes que por el método, que consistía en reunir experiencias, clasificarlas lo mejor posible y transmitirlas a los alumnos (Feyerabend, 2003, pp. 10 y 11). Francis Bacon consideró que lo esperable de un médico compasivo es que aplique su arte y celo para desembarazar al muriente de la vida del modo más suave y simple (Manzini, 1997, p. 135). 
Durante mucho tiempo no estuvo permitida en Argentina la eutanasia pasiva; de hecho, fue negada y aún lo es, lo que puede percibirse en fallos como el de la Corte Suprema de Justicia de Bs. As. "S., M. de C. s/ insania". En este caso, el esposo de la mujer hace referencia a la calidad de vida, es decir, al componente que tiene la frase tan usada de "muerte digna". La muerte se vuelve digna cuando al final de la vida se llega sin sufrir ni degradar la condición humana, en gran parte medicalizando, es decir, manteniendo a quien debe irse. Se complejiza la situación si al mero sostenimiento biológico de un cuerpo se le suma también el aspecto cualitativo, digno, humano. Señala el esposo: "'[...] es una herida abierta... una degradación de ella misma..., que no permite tener paz... como si un objeto no permitiese nunca una cicatrización... vive degradándose..." (consid. 5 del voto del juez Negri).

A pesar de su voto contrario al pedido del marido, el juez Hitters menciona la concepción que llama "romanista", que implica una idea paternalista de la medicina (consid. 3), es decir, una actitud medicalizadora, por cuanto puede decirse que el fundamento cultural de la medicalización se encuentra en la irrefrenable actitud tan hispánica (Weber, 1998) de decir a los demás lo que hay que hacer. En este caso, la medicina no es más que un asunto de una filosofía paternalista, que tiene sus aplicaciones en la política, con la llamada "democracia", que de gobierno del pueblo tiene poco, si solo se percibe lo que ¿elije? el cuerpo electoral cada dos años y la escasa rendición de cuentas, y ausencia de claridad y acceso a las plataformas electorales. Aquí también se percibe la complejidad del problema si la medicina se relaciona con la política, la cultura y la filosofía. El creador del pensamiento complejo visualiza el relacionamien- to de la política con aspectos claves de la vida/muerte. Así,

[...] con la posibilidad de modificar la forma de transmisión del patrimonio hereditario y el propio patrimonio, la naturaleza humana y la naturaleza de la sociedad entran en la problemática política: vivir, nacer y morir ya están instalados en el campo de la política. Las perturbaciones que afecten las nociones de padre, madre, niño, masculino, femenino, es decir, lo que había de fundamental en la organización de la familia y de la sociedad, requieren normas políticas. La noción de ser humano, que ha llegado a ser modificable por manipulaciones, corre el peligro de ser normalizada por un poder político que disponga del poder de manipular el poder de manipulación. (Morin, y Kern, 2006, p. 159)

El juez Hitters expresa en sus consideraciones la posición juridizadora, cuando señala que "[...] aún en caso de duda, siempre debe estarse por la solución más favorable a su prolongación (o subsistencia)" (consid. 3). Se refiere a la vida. En caso de duda, si la hubiera, debe estarse a favor de la calidad de la vida, no a favor del mantenimiento sin sentido de esta, si tal fue la intención del paciente. La introducción de la rama del derecho de la salud plantea otra visión al poner como eje de la discusión la salud en lugar de la vida, es decir, las condiciones de salud en las que se vive, frente a términos absolutos como la vida, siempre considerada "sagrada". El juez Roncoroni también adopta una postura juridizadora, puesto que ante la duda se pronuncia por mantener la vida: "[...] no queda otra conducta que continuar con los tratamientos" (consid. 6). Si en el caso no se pudo acceder a la voluntad del paciente, se la pudo reconstruir, o 
asumir la posibilidad de la sustitución, en su representante legal o en la familia. La expresión "no queda otra" es muy similar a cuando se dice "es lo que hay". En realidad, se quiere lo que hay, porque si no se haría otra cosa. La juridización se puede dar no solamente por actitudes comisivas, sino también por omisiones. El juez Roncoroni estaría inmerso en la judicialización, es decir, un caso de juridización judicial por omisión, cuando señala: "[...] no encuentro en nuestra legislación una norma que me permita acoger su petición" (consid. 8). Cuando no había una ley que protegiera los bosques nativos, ¿había que permitir las talas para lograr terrenos aptos para plantación de soja? Dice Sanpedro (2005): "se puede lavar la responsabilidad diciendo que eso es asunto a resolver por el legislador, y mientras la norma nos favorezca, lo más cómodo es cumplir con el deber" (p. 202). Hay que lograr la justicia, para lo cual las normas son un medio, pero no el fin en sí mismo. "Pero el juez, el fiscal y todo ser bien nacido, tiene el deber moral de ser justo. Y la justicia es, antes que la ley, razón ética y humana" (Sanpedro, 2005, p. 201). Lo que debe lograrse aquí es la salud de la persona, no cumplir normas. Esta juridización es parecida a la sistémica que defino como aquella que proviene de todos los estamentos estatales, como se da con el tema de las drogas. El juez Negri introduce la cuestión importante de los legitimados para intervenir en la decisión eutanásica, en tanto el juez anterior centró la atención en el consentimiento de la persona que se encuentra como paciente, pero la salud no puede entenderse de manera individual. Cuando hay una enfermedad, no solo la padece el individuo, sino también su familia, su localidad y la comunidad toda. La familia sufre por no poder hacer el duelo, y la comunidad también por verse perjudicada en la falta de recursos sanitarios que se siguen empleando en quien no mejora su estado de sa- lud. El juez se refiere solamente al círculo familiar, que merece llevar "[...] un final a una situación patética que se anticipa irreversible y dar término, simultáneamente, a la congoja que la misma provoca a su marido y a sus hijos" (consid. 1). El magistrado es más explícito aun cuando señala: "[...] es un problema psicológico... 'para él y para sus hijos su vida, advirtiendo que '...ella merece morir dignamente y no permanecer, en forma indefinida y subhumana (sic) en la vida vegetativa...' [...]" (consid. 4). Se ha dicho que el uso de nuevas tecnologías como la ventilación mecánica o el masaje de resucitación cardio-pulmonar creó formas de vida híbridas donde el estado provisorio deviene crónico, y en ese estado las personas no pueden expresarse ni reflexionar sobre ellas mismas, pierden su estatus de sujetos y devienen objetos de cuidados y de la ciencia; ni muertos ni vivos, ellas reducen la significación de la vida y la muerte a nada (Jolicorps, 2007, p. 4). En una de sus cartas, Ramón Sanpedro (2005) dice: "Y no me dejan ser ni muerto ni vivo [...]" (p. 24).

El juez expresa: "no puedo, como juez de derecho, acceder a la petición de dejar morir de hambre y sed [...]" (consid. 14). Recorriendo el catálogo de falacias, podría crearse una que se tratara de "apelación al sentimiento", como cuando una persona llora en una discusión, para apelar así a la lástima.

En particular, quisiera poner de relieve que la administración de agua y alimento, aunque se lleve a cabo por vías artificiales, representa siempre un medio natural de conservación de la vida, no un acto médico. Por tanto, su uso se debe considerar, en principio, ordinario y proporcionado, $y$ como tal moralmente obligatorio, en la medida y hasta que demuestre alcanzar su finalidad propia, que en este caso consiste en 
proporcionar alimento al paciente $\mathrm{y}$ alivio

a sus sufrimientos. (Juan Pablo II, 2004)

Hay que tener en cuenta que no puede abandonarse a quien ya está abandonado. ¿Cuál es, entonces, el sentido de dignidad en tratar de curar lo incurable? ¿Cómo puede ocurrírsele a alguien plantear la muerte por inanición cuando no hay ninguna hipótesis de sentir ni sed ni hambre en quien ha perdido la conciencia y la capacidad de sentir para siempre? (Maglio, 2012).

La apelación al sentimiento parece ingenua, tierna y misericordiosa, y la confusión en la que sumerge confirma su categorización como falacia. ${ }^{5}$ Pero en el fondo encierra la voluntad de dominar la vida de las personas y evitar la muerte digna, que es el anverso de una vida digna. Dice Sanpedro (2005): “[...] mantener en los individuos el temor psicológico al castigo por actuar libremente llega a ser la forma más eficaz de dominarlos. Y también la de destruirlos" (p. 148).

Sanpedro (2005), además, brinda una excelente lección acerca del papel del juez, que no llamo autómata, sino complejo:

[...] me parece que la función de los jueces tiene que ser algo más que la de aplicarle códigos al rebaño como un mudo y fiel guardián que defiende los intereses de su degenerado amo. Cuando un juez guarda silencio ante una ley obviamente hipócrita, y por tanto injusta, en esa sociedad no puede haber nobleza ni bondad. Si la justicia es la exigencia de una conducta ética

5 "[...] una falacia es una maniobra verbal destinada a conseguir que alguien acepte una afirmación $\mathrm{u}$ obedezca una orden por motivos que no son buenas razones" (Comesaña, 2001, p. 53). respetuosa, la función del juez debe ser la de maestro más que la de vigilante. (p. 253)

La Ley 26742, que reforma la Ley de Derechos del Paciente, es favorable a la ausencia de juridización de la salud, por cuanto promueve un obstáculo al tratamiento médico que sea desproporcionado. Dice el artículo 5, inc. g:

El derecho que le asiste en caso de padecer una enfermedad irreversible, incurable, o cuando se encuentre en estadio terminal, o haya sufrido lesiones que lo coloquen en igual situación, en cuanto al rechazo de procedimientos quirúrgicos, de hidratación, alimentación, de reanimación artificial o al retiro de medidas de soporte vital, cuando sean extraordinarios o desproporcionados en relación con las perspectivas de mejoría, o que produzcan sufrimiento desmesurado, también del derecho de rechazar procedimientos de hidratación y alimentación cuando los mismos produzcan como único efecto la prolongación en el tiempo de ese estadio terminal irreversible e incurable.

No obstante, la ley de "muerte digna" es juridizadora porque no permite el suicidio asistido, ${ }^{6}$ aunque sí el rechazo a los tratamientos médicos, lo que se dio en el caso "Jorge Albarracini Nieves", fallado el 1.6.2012 por la Corte Suprema de Justicia de la Nación (CSJN), que trataba de un testigo de Jehová, amparado en su derecho a rechazar una transfusión sanguínea. Dice la ley: "En todos los casos la negativa o el rechazo de los procedimientos mencionados no significará la interrupción de aquellas medidas y acciones para el ade-

6 Tampoco lo permite el artículo 83 del CP.: "Será reprimido con prisión de uno a cuatro años, el que instigare a otro al suicidio o le ayudare a cometerlo, si el suicidio se hubiese tentado o consumado". 
cuado control y alivio del sufrimiento del paciente" (artículo 5, g).

El fallo del tribunal superior de Neuquén en el caso "D. M. A. s/ declaración de incapacidad, del 19.4.2013, trata el caso de Marcelo Diez. La sentencia señala, luego de mencionar el fallo Albarracini sobre rechazo de tratamientos, e interpretando la ley de muerte digna, que la idea que la inspira es evitar la judicialización, una especie de juridización de la salud: "[...] en tan penosas circunstancias no se susciten dudas respecto del proceder legal, las que devienen en conflictos que desplazan la relación médico-paciente al ámbito judicial, lo cual necesariamente rompe ese vínculo esencial" (pto. III). El tribunal vuelve sobre el punto al señalar: "[...] la nueva normativa de Derechos del Paciente, establece un procedimiento que no requiere intervención judicial para la petición efectuada por las hermanas de M. A. D." (consid. IV). Posteriormente, expresa el lugar donde todo aquello debe resolverse: "[...] estas penosas situaciones no deben desbordar el ámbito íntimo del paciente y/o de su familia y el médico tratante". Incluso da cuenta de otro caso de juridización, utilizando dicho término, del acto médico que es el caso del aborto no punible, ya que a pesar de su reconocimiento legal en el código de fondo, los profesionales de la salud se niegan a aceptarlo, como ocurrió en el caso "F. A. L." (consid. IV). Uno de los jueces que vota, en particular, señala algo que es común a estos problemas biojurídicos, y es el hecho de que las normas que reconocen derechos solo los permiten a los ciudadanos, pero no los imponen. Lo que ha ocurrido con el divorcio, la fertilización asistida, la muerte digna por este caso, etc., cuando desde asociaciones católicas, como Portal de Belén, Asociación Civil Cristo Sacerdote, Asociación Civil Mujeres por la Vida, etc., se pretende que toda la población argentina adopte postulados de la religión católica: la prohibición de la pastilla del día después, la prohibición del arte crítico de León Ferrari, el programa de salud sexual y procreación responsable, respectivamente.

Que una persona pueda contar con la posibilidad de las directivas anticipadas de salud significa una intervención del derecho (artículo 11, Ley 267429) para posibilitar la autonomía de la persona. Porque antes, quienes dirigían la salud de las personas eran los médicos $y$, en el mejor de los casos, los familiares que tenían consciencia del problema de salud y una buena relación con el equipo sanitario.

También puede haber juridización doctrinaria, que se da cuando la academia, los autores prestigiosos en el ámbito de la medicina o el derecho tratan temas personales como patológicos o jurídicos.

Ante la ausencia de directivas anticipadas, cabe preguntarse si hay que reconstruir la voluntad del paciente, en el sentido de averiguar lo que habría querido de encontrarse en una situación de eutanasia y de imposibilidad de manifestar su voluntad, o atender la voluntad de su círculo íntimo, no necesariamente identificado con los parientes por consanguinidad o afinidad. La Ley 26742 hace referencia, en general, a que todo tratamiento médico-sanitario requiere el previo consentimiento informado del paciente. Luego de lo cual señala:

En el supuesto de incapacidad del paciente, o imposibilidad de brindar el consentimiento informado a causa de su estado físico o psíquico, el mismo podrá ser dado por las personas mencionadas en el artículo 21 de la Ley 24.193, con los requisitos y con el orden de prelación allí establecido. 
Sin perjuicio de la aplicación del párrafo anterior, deberá garantizarse que el paciente en la medida de sus posibilidades, participe en la toma de decisiones a lo largo del proceso sanitario.

Esto significa que la intención del legislador parece que se dirige al hecho de que se respete la intención del paciente. Esto habilitaría al juez o al comité, según el caso, a revisar la decisión de los familiares, en tanto contradigan algún elemento probatorio destinado a construir la voluntad del paciente. En efecto, de lo que se trata no es de aceptar la voluntad sustituta de los familiares, sino de que ellos cumplan la función de señalar lo que el paciente hubiera querido. Así lo señala la CSJN al fallar el caso "Diez": "[...] no deciden ni en el lugar' del paciente ni 'por' el paciente sino comunicando su voluntad" (consid. 22). De manera similar a lo que ocurre con la interpretación de las normas, no se trata de que el encargado de su funcionamiento elija el sentido que mejor le plazca, incluso diciendo seguir pautas de justicia o la "voluntad de la ley"; de lo que se trata es de ver cuál fue la intención del autor de la norma para evidenciarlo, si de algo sirve "desentrañar el sentido" de algo, esto es, de la norma por interpretar. Luego, el intérprete deberá decir si está en desacuerdo con la norma rectamente interpretada, asumiendo el costo de haber elaborado una norma distinta, por razones de carencia de justicia. Así como Goldschmidt (1987) previó las "manifestaciones extranormativas" (p. 258), que son aquellos elementos distintos de la norma que ayudan a interpretarla, como la exposición de motivos, considerandos debates parlamentarios, la ley anterior, etc., también puede haber "manifestaciones vitales" del paciente: una conversación, un libro, un diario, una reunión familiar, etc. Todo esto serviría para reconstruir su historia. El juez Roncoroni, en el caso "S., M. de C., extrema los recaudos a la hora de la reconstrucción:

Es menester que la historia narrada por la testigo nos ponga frente a una persona que, buceando en lo más profundo de su alma y de su razón y llevada a la viva representación de su posible situación terminal, nos brinde una reflexión harto ponderada y madura sobre su propio destino. Una suerte de confesión en la que con verdadero compromiso racional y emotivo -que alcanza el tono del ruego, del mandato o del mensaje o prescripción a cumplir en el futuro- se proclama esa voluntad terminal. (consid. 6)

Lo anterior asimila la manifestación extravolitiva del paciente con su manifestación de voluntad, de ahí que sea injusta la parte del artículo 3, por cuanto habla de que "el mismo podrá ser dado". Lo que hay que hacer no es dar, sino "reconstruir" el consentimiento. La familia es un medio para la reconstrucción, ya que se supone que es la que tiene contacto con el paciente, pero se sabe que ello no siempre es así, y esto se refuerza con la última frase del artículo 3: "Sin perjuicio de la aplicación del párrafo anterior [orden de familiares de la ley de trasplantes], deberá garantizarse que el paciente, en la medida de sus posibilidades, participe en la toma de decisiones a lo largo del proceso sanitario".

De la lectura del artículo 21 de la Ley 24193 de trasplante de órganos, surge el orden con el cual los familiares deberán manifestar la voluntad reconstructiva. En dicho artículo, reforzando mi tesis, se dice:

\footnotetext{
[...] deberá requerirse de las siguientes personas, en el orden en que se las enumera siempre que estuviesen en pleno uso de sus facultades mentales, testimonio sobre la última
} 
voluntad del causante, respecto a la ablación de sus órganos y/o la finalidad de la misma.

No se pide lo que ellos quieren, sino lo que el paciente hubiera querido, lo que no obsta a que se reclame en justicia la participación en la situación de salud por derecho propio. Recuérdese que la Organización Mundial de la Salud ha ampliado el concepto de salud a la completa situación de bienestar bio-psico-social. Desde una perspectiva gestáltica, no hay una persona enferma, sino un ámbito donde hay un enfermo.

[...] la realidad no es un simple conjunto de hechos elementales, sino que consiste en unidades donde las partes no existen por sí mismas, donde cada parte apunta más allá de sí misma implicando un vasto todo. Los hechos y el significado cesan de ser dos conceptos pertenecientes a dominios diferentes, ya que un hecho es siempre un hecho dentro de un todo intrínsecamente coherente. [...] La solución debe venir del todo. [...] Se ha dicho: El todo es más que la suma de sus partes. Más correcto es decir que el todo es otra cosa que la suma de sus partes, porque la suma es un procedimiento sin sentido, mientras que la relación todo-parte está llena de sentido. (Koffka, 1953, pp. 210 y 211)

Dicha idea es compartida por el pensamiento complejo, que señala:

\footnotetext{
La idea de totalidad [...] a menudo había salido a la superficie en la historia de la filosofía, se había expandido en la filosofía romántica y sobre todo en Hegel. Surge a veces en las ciencias contemporáneas así como en la teoría de la forma o Gestalt (Morin, 1993, p. 129)
}

\section{Aspecto jurístico-dikelógico}

Edgar Morin (2006b) planteaba la necesidad de unir en la ciencia los juicios de hecho con los juicios de valor, e incluir la ética en toda disciplina científica, por cuanto "[...] la época en que los juicios de valor no tenían que interferir con la actividad científica está cerrada" (Morin, 2006c, p. 79). Por ello es importante que el derecho no sea disociado de la justicia, sino que esta sea un momento o elemento de su análisis (Galati, 2012).

Ramón Sanpedro (2005) señala: "Allí donde no predomine la razón pura sobre la ley, sobrevivirá eternamente la injusticia" (p. 201). La mera separación que condena la complejidad es captada en la problemática: "[...] quienes no desean modificar la norma que protege su personal interés o de grupo, afirman que eso es filosofía" (Sanpedro, 2005, p. 201). En efecto, todo es filosofía. No es que las irracionalidades, mezquindades e hipocresías tengan algo personal con la eutanasia; también la ley ha sido injusta en otros ámbitos: al prohibir el divorcio y el casamiento entre homosexuales, al separar a los herederos según estén o no posesos de un vínculo "legal" o "legítimo", al humillar a la mujer en relación con el varón en su capacidad civil, electoral, laboral y gestacional, etc.

El fundamento de la bioética, si se toma desde su origen, fue acompañar a la medicina en el abordaje de los problemas de la vida y la salud, porque ellos no se agotan en sus aspectos meramente biológicos. ${ }^{7}$ El espíritu se alimenta también de las creencias que tiene la persona y no todas permiten lo

7 En el mismo sentido, se asocia la enfermería al cuidado del paciente, procurando su salud, con una aproximación bio-psico-social del hombre, y la medicina al tratamiento de la enfermedad, con una aproximación biológica (Jolicorps, 2007, p. 7). 
mismo, de ahí que sea válida la conducta que, no perjudicando a los demás, tiene su postura ante la vida y el sufrimiento. "La calidad de la vida consiste en una conformidad placentera, una percepción armónica del cuerpo y de la mente con el todo al que están condicionados y sujetos los sentimientos personales" (Sanpedro, 2005, p. 58). El criterio del perjuicio es el que empleó la CSJN en el caso "Arriola" del 25.8.2009, para despenalizar el consumo de marihuana en ese caso, precisamente porque lo entiende personal, sin impacto en el público, y de esta manera se vuelve una conducta autorreferencial y, en ese sentido, gobernada por la propia persona. Al respecto, dice Sanpedro (2005):

\section{Justificar sufrimientos irremediables por el interés de alguien que no sea el desafor- tunado ser humano que los padece es crear el infierno para qué diablos y diablillos dis- fruten con el espectáculo de los condena- dos, mientras filosofan gravemente sobre el sentido del dolor. (p. 255)}

He aquí la esencia de la medicalización, que apunta en este caso a la despersonalización, y que contraria así otra idea clave del pensamiento complejo, la singularidad:

\section{[...] hemos visto que el paradigma 'no hay ciencia más que de lo general', que excluía toda individualidad y toda singularidad, debe ser radicalmente superado: el objeto primero de toda ciencia, el mundo, es sin- gular en su origen, en su globalidad, en sus desarrollos, y esto es lo que fundamenta la generalidad de las Leyes de la Naturaleza, leyes universales de nuestro Universo sin- gular [...]. (Morin, 1993, p. 428)}

Cabe analizar dónde la medicina plantea su actuación de manera injustificada. Cuando se hace referencia a la eutanasia pasiva, pareciera que ella va contra la medicalización, justamente porque si se hiciera ese algo para intervenir en la vida de la persona -aunque tal vez para mejorar temporariamente su salud- no afectaría el estado de base que es la irreversibilidad de la enfermedad y de ahí la omisión, como el rechazo a realizar determinadas prácticas, como la reanimación, la traqueotomía, etc.

En la eutanasia activa, la medicina interviene para eliminar el dolor, pero causa la muerte de la persona. Su intervención es legítima, pero es un recurso extremo, tal vez justificado cuando la tecnología médica no pueda deshacerse del dolor, el presupuesto principal de la existencia de la eutanasia. Hoy en día, los cuidados paliativos, que ayudan a calmar los padecimientos vinculados al final de la vida, hacen innecesaria la focalización en la muerte. Hay que tender, no obstante, a que los cuidados paliativos no devengan en obstinación o encarnizamiento terapéutico, porque lo que es fácil de distinguir en el ámbito de la teorización es difícil en el campo de los hechos. La vida es un océano de interacciones que se resiste a las clasificaciones y etiquetamientos. Justamente, en este punto se propone la tarea de la diferenciación de Jorge Manzini (1997), que señala:
[...] ocurre muchas veces que el control de los síntomas que provocan gran sufrimien- to al paciente, especialmente el dolor y la disnea [dificultad de respirar], no puede lo- grarse sin provocar sedación, aún sueño y depresión respiratoria; que los pacientes en ese estado no ingerirán líquidos ni alimen- tos, y que su vida se acortará. (p. 138)

Aquí se ve cómo la línea divisoria entre cuidados paliativos y eutanasia activa indirecta, 
incluso entre cuidados paliativos y eutanasia pasiva, es difícil de trazar.

La dimensión de la justicia, autónoma pero no separada de la normativa, precisamente porque el derecho es un proceso complejo en el cual cabe percibir distintos aspectos -todos de un mismo fenómeno-, permite la crítica de la normatividad sin dejar de estar en el Derecho. La justicia es también compleja como el derecho, lo que se traduce en distintos aspectos dentro de ella. Sus elementos principales son la intervención y la autonomía, lo cual tiene su aplicación en el campo de la salud, por cuanto hay que lograr un equilibrio entre el poder del médico y el derecho del paciente. Precisamente, el derecho de la salud nace para equilibrar esos elementos de la relación, para proteger al paciente del inmemorial poder del médico y para procurar la salud. Cabe preguntarse si hay que abstenerse para respetar la autonomía del paciente que quiere morir y ayudarlo a suicidarse, como en el caso de Ramón Sanpedro, si hay que respetar la decisión de la familia, que quiere dejar ir al enfermo en situación de eutanasia, o si van a intervenir en dicha "vida" para mantenerla. Cuando Goldschmidt definía el supremo principio de justicia, no apuntaba a la felicidad del pueblo, sino a garantizar a cada uno un espacio de libertad, para que sea el individuo quien elija cómo ser feliz según su cosmovisión. ${ }^{8}$

8 " [...] la justicia no tiene la misión de desplegar la personalidad del individuo, sino la de proporcionarle la libertad necesaria para desplegarla. Dentro de este espacio de libertad, cada cual debe desarrollarse según su propia manera de ser [...]" (Goldschmidt, 1958, p. 231). De lo cual se sigue que "[...] ninguna adjudicación debe estrechar excesivamente el ámbito de libertad del hombre [...]" (p. 399).

\section{Conclusión}

El horizonte filosófico de la eutanasia implica la posibilidad de poner fin a esa vida, contra lo cual va la medicalización de ella, por cuanto se vale de la tecnología médica creyendo que lo que queda de ella puede ser asemejado a una vida plena. La perspectiva que se ha adoptado en este trabajo, como decisión teórica, es que la medicalización de la vida siempre es inapropiada, inadecuada, porque si la medicina interviene cuando debe, no medicaliza, simplemente hace todo lo posible para mejorar la salud del paciente.

En el trabajo se desarrolla la idea de medicalización en el ámbito de la eutanasia y se realiza un tratamiento jurídico complejo de la cuestión, en el triple abordaje sociológico, normológico y dikelógico. Si bien dicho tratamiento jurídico ya es complejo de por sí, por la multidimensionalidad del análisis, se relaciona la medicalización de la eutanasia con algunas ideas específicas del pensamiento complejo de Morin. Además, se conceptualiza la idea de juridización y se categorizan sus despliegues.

La bioética nació para cuestionar la medicina, justamente porque esta se basa en el poder del médico para decidir sobre la vida ajena, así como en otro tiempo los que decidían eran los sacerdotes. La Modernidad significó lo nuevo, lo reformador, como ocurrió cuando se reafirmaba el sujeto audaz de Cristóbal Colón que "descubría" nuevas tierras, el hombre que se soltaba a cuestionar los poderes hegemónicos, que se embarcaba en la empresa capitalista y que fundaba el conocimiento en la experiencia. ¿Qué es lo nuevo, lo moderno actualmente? Hoy, en el siglo XXI, el poder apunta a retornar al individuo. Lo tuvo la Iglesia, lo tuvo la medicina, lo tiene el mercado, ¿lo tendrá el individuo? 
Decía Sanpedro: “¿Por qué morir?, porque todo viaje tiene su hora de partida, y todo el que va de viaje tiene el privilegio, y el derecho, de escoger el mejor día de salida" (Sanpedro, 2005, p. 89). No hay que olvidar entonces que la bioética nació para libertar al individuo, y que hoy se hace compleja para mostrarnos un camino hacia dicha meta.

\section{Referencias}

Coic G., A. (2008). El arte de la medicina y el médico humanista. En O. Garay (Coord.), Bioética en Medicina (pp. 509-529). Buenos Aires: Ad-Hoc.

Comesaña, J. M. (2001). Lógica informal, falacias y argumentos filosóficos. Buenos Aires: Eudeba.

Egúsquiza Pozo, V. (2005). Dilucidación de la cientificidad de la enfermería (Tesis de maestría). Lima, Universidad Nacional Mayor de San Marcos.

Epele, M. (2008). Usos y abusos de la medicalización en el consumo de drogas: sobre economías, políticas y derechos. En A. Cannellotto y E. Luchtenberg (Coords.), Medicalización y sociedad. Lecturas críticas sobre un fenómeno en expansión. Recuperado de http://scripts. minplan.gob.ar/octopus/archivos. php?file $=4271$

Feyerabend, P. (2003). La science en tant qu'art (Trad. F. Périgaut). Paris: Albin Michel.

Foucault, M. (1996). La vida de los hombres infames (Trads. J. Varela y F. Álvarez-Uría). La Plata: Altamira.

Galati, E. (2012). Introducción al pensamiento jurídico complejo. La teoría trialista del mundo jurídico y el pensamiento complejo de Edgar Morin. Revista de la Facultad de Derecho, (20), 157-215.

Goldschmidt, W. (1987). Introducción filosófica al Derecho (6ta. ed.). Buenos Aires: Depalma.

Goldschmidt, W. (1958). La ciencia de la justicia (Dikelogía) (2da. Ed.). Madrid: Aguilar.

Gutiérrez-Samperio, C. (2001). La bioética ante la muerte. Gaceta Médica de México, 137(3), 269-276.

Illich, I. (1975). Némesis médica. La expropiación de la salud. Barcelona: Barral.

Jolicorps, A. (2007). L'assistance au suicide en Suisse: de la légitimation à la pratique Ethnographie de l'association EXIT ADMD (Tesis de grado). Université de Neuchâtel Suiza.

Juan Pablo II (2004). Discurso a los participantes en un congreso sobre "tratamientos de mantenimiento vital y estado vegetativo". Recuperado de http://www. vatican.va/holy_father/john_paul_ii/ speeches/2004/march/documents/hf jp-ii_spe_20040320_congress-fiamc_ sp.html

Koffka, K. (1953). Principios de psicología de la forma. Buenos Aires: Paidós.

Maglio, I. (2012). Dejen ir a Marcelo en paz. Recuperado de http://www.rionegro. com.ar/diario/dejen-ir-a-marcelo-enpaz-943988-9539-nota.aspx

Mainetti, J. (2006). La medicalización de la vida. Electroneurobiología, 14(3), 71-89.

Manzini, J. (1997). Bioética paliativa. La Plata: Quirón. 
Morin, E. (2007). Articular los saberes ¿Qué saberes enseñar en las escuelas? ( $2^{\mathrm{a}}$ Ed., Trad. de Geneviève de Mahieu). Buenos Aires: Ediciones Universidad del Salvador.

Morin, E. (1984). Ciencia con consciencia (Trad. A. Sánchez). Barcelona: Anthropos.

Morin, E. (1993). El Método 1. La naturaleza de la naturaleza (Trad. A. Sánchez y D. Sánchez García). Madrid: Cátedra.

Morin, E. (2006b). El Método 4. Las ideas. Su hábitat, su vida, sus costumbres. (Trad. A. Sánchez). Madrid: Cátedra.

Morin, E. (2006a). Breve historia de la barbarie en Occidente (Trad. A. Grieco). Buenos Aires: Paidós.

Morin, E. (2006c). El Método 6. Ética. (Trad. A. Sánchez). Madrid: Cátedra.
Morin, E. (2005). Introducción al pensamiento complejo (Trad. M. Pakman). Barcelona: Ghedisa.

Morin, E., Ciurana, R. y Motta, R. (2002). Educar en la era planetaria. Valladolid: Unesco, Universidad de Valladolid.

Morin, E. y Hessel, S. (2013). El camino de la esperanza. Una llamada a la movilización cívica (Trad. R. Alapont). Buenos Aires: Paidós.

Morin, E. y Kern, A. B, (2006). Tierra patria (Trad. R. Figueira). Buenos Aires: Nueva Visión.

Moynihan, R. y Cassels, A. (2006). Medicamentos que nos enferman e industrias farmacéuticas que nos convierten en pacientes (Trad. H. Montagut). Buenos Aires: Atlántida. 